\title{
Does the Impact of Smoking on Coronary Heart Disease Differ by Low-Density Lipoprotein Cholesterol Level? The Atherosclerosis Risk in Communities (ARIC) Study
}

\author{
Atsushi Hozawa, MD*,**; Aaron R. Folsom, MD**; A. Richey Sharrett, MD \\ Thomas J Payne, $\mathrm{PhD}^{\dagger+}$; Lloyd E. Chambless, $\mathrm{PhD}$
}

\begin{abstract}
Background The association of smoking with coronary heart disease (CHD) occurrence has been reported to be weaker for populations with lower plasma cholesterol levels. Recent studies suggest that low-density lipoprotein cholesterol (LDL-C) and smoking contribute to different stages of atherosclerosis, so the present study was designed to test the hypothersis that smoking is a stronger risk factor for CHD when LDL-C is high.

Methods and Results The study group of 13,410 middle-aged adults who were initially free of stroke and CHD were followed and over 13.3 years there were 932 incident CHD events. Tests for multiplicative interaction were performed using proportional hazards models. Both smoking and increased LDL-C were risk factors for $\mathrm{CHD}$ incidence. The relative hazard (RH) of CHD in relation to smoking tended to be larger among higher LDL$\mathrm{C}$ categories compared with lower LDL-C categories. For example, when the participants were dichotomized into 4 categories, using smoking $\geq 15$ cigarettes per day and LDL-C $\geq 130 \mathrm{mg} / \mathrm{dl}$ as cutoffs, those with high LDL-C and heavier cigarette smoking showed a very high RH of CHD $(\mathrm{RH}=2.81)$ compared with that expected from the product of the RHs of high LDL-C $(\mathrm{RH}=1.15)$ only $\times$ heavy smoking only $(\mathrm{RH}=1.71)(\mathrm{p}$ for interaction $=0.04)$. Conclusions These results suggest positive multiplicative interactions between smoking and LDL-C for CHD incidence. (Circ J 2006; 70: 1105-1110)
\end{abstract}

Key Words: Coronary heart disease; Interactions; LDL-cholesterol; Prospective studies; Smoking

$\sim$ ig igarette smoking is a strong risk factor for coronary heart disease (CHD) incidence, although some epidemiological studies suggest that the strength of the association of smoking with CHD incidence may vary among populations. Smoking is a weaker risk factors in Asian countries compared with Western countries 2,3 and the Seven Countries Study showed that the relationship of smoking with CHD incidence was weaker in southern Europe than in Northern Europe and Yugoslavia4 This geographical difference in the effect of smoking with CHD might be explained by ethnic, cultural or environmental factors; for example, the level of total cholesterol (TC) or low-density lipoprotein cholesterol (LDL-C). People who live in Asia and in southern Europe at the time of the early follow-up of the Seven Countries Study had low average total and LDL-C levels? ${ }^{2-4}$

Recent studies comparing the contribution of cigarette smoking or LDL-C suggest that LDL-C is more important for early atheroma formation, whereas smoking affects the

(Received April 12, 2006; revised manuscript received June 1, 2006; accepted June 28, 2006)

*Division of Epidemiology and Community Health, School of Public Health, University of Minnesota, Minneapolis, MN, USA, **Department of Public Health and Forensic Medicine, Division of Epidemiology, Tohoku University Graduate School of Medicine, Sendai, Japan, tDepartment of Epidemiology, Johns Hopkins University, Bloomberg School of Public Health, Baltimore, MD, ${ }^{\dagger}$ Department of Medicine and ACT Center, University of Mississippi Medical Center, Jackson, MS and Department of Biostatistics, University of North Carolina, Chapel Hill, NC, USA

Mailing address: Atsushi Hozawa, MD, Department of Health Science, Shiga University of Medical Science, Setatsukinowa-cho, Otsu 520-2192, Japan. E-mail: ahozawa@ @elle.shiga-med.ac.jp later stages of atherosclerosis 5,6 These findings suggest that smoking may depend on elevated LDL-C levels to maximize its effect on CHD risk.

Thus, both international comparison and biological considerations suggest that smoking is a weaker CHD risk factor when LDL-C is low, and a stronger risk factor when LDL-C is high. However, epidemiological studies to date have failed to find clear differences in the relative risk of smoking for CHD across TC or LDL-C levels ${ }^{7-9}$

We compared the relative hazards (RH) of CHD in relation to cigarette smoking status and cigarette-years of smoking in participants with lower vs higher LDL-C levels using the Atherosclerosis Risk in Communities (ARIC) Study cohort.

\section{Methods}

Study Design and Subjects

The ARIC Study is a prospective study of atherosclerotic disease in 4 US communities: (1) Forsyth County, North Carolina, (2) Jackson, Mississippi, (3) Washington County, Maryland and (4) the northwest suburbs of Minneapolis, Minnesota $!^{10}$ The cohort comprised, at baseline in 1987-89, 15,792 men and women aged 45-64 years who were selected by list or area probability sampling. Only AfricanAmericans were recruited in the Jackson study center. The baseline home interview assessed participants' sociodemographic characteristics, smoking and alcohol-drinking habits, medication use, reproductive history, and personal history of diseases. A clinic examination included measurement of various risk factors and cardiovascular conditions, B-mode ultrasound examinations of the carotid and 
Table 1 Prevalences or Mean Values of Baseline Participant Characteristics According to Approximate Quartiles of Plasma LDL-C and Smoking Status: Atherosclerosis Risk in Communities (ARIC) Study, 1987-1989

\begin{tabular}{|c|c|c|c|c|c|c|c|c|c|c|}
\hline & \multicolumn{6}{|c|}{ Serum $L D L-C(m g / d l)$} & \multicolumn{4}{|c|}{ Smoking status } \\
\hline & $<99$ & $100-129$ & $130-159$ & $160-189$ & $\geq 190$ & $p$-value & Current & Former & Never & $p$-value \\
\hline$N$ & 2,175 & 3,887 & 3,393 & 2,231 & 1,184 & & 3,392 & 4,257 & 5,761 & \\
\hline Age (years) (Mean) & 52.7 & 53.5 & 54.3 & 54.9 & 55.2 & $<0.01$ & 53.4 & 54.6 & 53.9 & $<0.01$ \\
\hline $\operatorname{Men}(\%)$ & 36.0 & 41.4 & 46.4 & 48.3 & 39.5 & $<0.01$ & 44.8 & 59.5 & 29.7 & $<0.01$ \\
\hline African-American (\%) & 29.1 & 24.2 & 22.8 & 23.7 & 32.5 & $<0.01$ & 28.7 & 18.8 & 27.9 & $<0.01$ \\
\hline Diabetes (Yes) (\%) & 8.1 & 8.0 & 9.9 & 10.0 & 12.9 & $<0.01$ & 8.4 & 9.1 & 10.1 & 0.03 \\
\hline \multicolumn{11}{|l|}{ Smoking } \\
\hline Current (\%) & 25.8 & 24.6 & 24.2 & 26.7 & 27.6 & 0.18 & 100.0 & 0.0 & 0.0 & $<0.01$ \\
\hline Former (\%) & 30.9 & 31.5 & 32.5 & 31.7 & 31.5 & & 0.0 & 100.0 & 0.0 & \\
\hline Never (\%) & 43.3 & 43.9 & 43.3 & 41.6 & 40.9 & & 0.0 & 0.0 & 100.0 & \\
\hline \multicolumn{11}{|l|}{ Education } \\
\hline Less than high-school (\%) & 20.8 & 20.3 & 21.7 & 23.6 & 26.4 & $<0.01$ & 28.8 & 20.2 & 19.1 & $<0.01$ \\
\hline High-school (\%) & 28.8 & 32.7 & 34.1 & 33.8 & 35.6 & & 34.1 & 30.1 & 34.3 & \\
\hline More than high-school (\%) & 50.3 & 46.9 & 44.0 & 42.5 & 38.0 & & 37.1 & 49.7 & 46.4 & \\
\hline Use of antihypertensive medication (\%) & 26.6 & 25.1 & 27.1 & 27.8 & 33.4 & $<0.01$ & 25.1 & 26.6 & 28.7 & $<0.01$ \\
\hline Systolic blood pressure ( $\mathrm{mmHg}$ ) (Mean) & 118.9 & 119.8 & 121.4 & 121.4 & 123.2 & $<0.01$ & 119.1 & 121.0 & 121.5 & $<0.01$ \\
\hline$L D L-C(m g / d l)(M e a n)$ & 82.5 & 116.0 & 144.3 & 172.8 & 214.1 & $<0.01$ & 138.1 & 136.9 & 136.4 & 0.11 \\
\hline$H D L-C(m g / d l)(M e a n)$ & 60.1 & 53.7 & 50.5 & 48.4 & 48.5 & $<0.01$ & 50.1 & 51.3 & 54.8 & $<0.01$ \\
\hline Triglyceride (mg/dl) (Mean) & 107.0 & 113.4 & 125.7 & 137.5 & 147.5 & $<0.01$ & 127.2 & 127.2 & 117.4 & $<0.01$ \\
\hline Ethanol intake (g/week) (Mean) & 49.1 & 42.3 & 40.1 & 39.9 & 38.1 & $<0.01$ & 66.1 & 52.8 & 19.8 & $<0.01$ \\
\hline Waist-to-hip ratio (Mean) & 0.90 & 0.92 & 0.93 & 0.94 & 0.94 & $<0.01$ & 0.92 & 0.94 & 0.91 & $<0.01$ \\
\hline Body mass index $\left(\mathrm{kg} / \mathrm{m}^{2}\right)($ Mean $)$ & 26.6 & 27.5 & 27.8 & 27.9 & 28.2 & $<0.01$ & 26.3 & 27.8 & 28.1 & $<0.01$ \\
\hline Sports index (Mean) & 2.4 & 2.4 & 2.5 & 2.5 & 2.4 & $<0.01$ & 2.3 & 2.6 & 2.4 & $<0.01$ \\
\hline
\end{tabular}

LDL-C, low-density lipoprotein cholesterol; HDL-C, high-density lipoprotein cholesterol.

p-value was assessed by ${ }^{2}$-test or analysis of variance as appropriate.

popliteal arteries, and a 12-lead electrocardiogram (ECG).

\section{Baseline Examination}

Methods for blood collection and processing in ARIC have been described in detail.1 ${ }^{1}$ Fasting plasma TC and triglycerides (TG) were measured by enzymatic methods, 12,13 high-density lipoprotein cholesterol (HDL-C) level was measured by the method of Warnick et al, ${ }^{14}$ and LDL-C concentration was calculated by the Friedewald formula! 15 The classification of LDL-C subgroups was that defined by the Executive Summary of The Third Report of The National Cholesterol Education Program (NCEP) Expert Panel on Detection, Evaluation, and Treatment of High Blood Cholesterol In Adults (Adult Treatment Panel III): that is, <100 mg/dl: Optimal; 100-129 mg/dl: Near optimal/ above optimal; 130-159 mg/dl: Borderline high; 160$189 \mathrm{mg} / \mathrm{dl}$ : High; and $\geq 190 \mathrm{mg} / \mathrm{dl}$ : Very high! 16 An intraindividual variability study demonstrated excellent shortterm repeatability in ARIC, with a reliability coefficients $\geq 0.85$ for LDL-C, HDL-C, and TG. ${ }^{17}$

Usual ethanol intake and smoking status (current, former, or never smokers) were assessed by interview! ${ }^{8}$ Smokers (current or former) were asked the average numbers of daily cigarettes smoked during the time they smoked. Cigarette-years of smoking were calculated as the average number of cigarettes per day multiplied by the number of years of smoking. Sitting blood pressures (BP) were measured 3 times using a random-zero sphygmomanometer after a 5-min rest. ${ }^{19}$ The mean of the last 2 measurements was used for analysis. Use of antihypertensive medications within the past 2 weeks of the baseline interview was selfreported 18 Serum glucose was measured by a hexokinase/ glucose-6-phosphate dehydrogenase method. Diabetes mellitus was defined as a fasting glucose $\geq 126 \mathrm{mg} / \mathrm{dl}$, or a reported history of physician-diagnosed diabetes, or current use of diabetes medication. Body weight was measured to the nearest pound and height was measured to the nearest centimeter. Body mass index (BMI) was computed as weight $(\mathrm{kg})$ divided by square of height $\left(\mathrm{m}^{2}\right)$. Waist-to-hip ratio (WHR), an indicator of fat distribution, was computed as the circumference of the waist (umbilical level, $\mathrm{cm}$ ) divided by the maximum circumference of the hips $(\mathrm{cm})$. The sports index, derived from the survey of Baecke et al,20 ranged from 1 (low) to 5 (high) for physical activity during leisure time.

Pre-existing CHD at baseline was defined by self-reported prior physician diagnosis of myocardial infarction (MI) or coronary revascularization, or by prevalent $\mathrm{MI}$ on the ARIC ECG ${ }^{21}$ Pre-existing stroke was defined by any self-reported prior physician diagnosis of stroke.

\section{Ascertainment of Incident CHD Events}

Interviewers contacted participants annually. All identified hospital records were reviewed and those suggesting CHD were abstracted for validation.$^{10,22}$ MI was validated using presenting signs and symptoms, peak cardiac enzyme levels, and Minnesota Coding 23 from up to 3 ECGs. Death certificates were also surveyed to identify CHD deaths. For validation of out-of-hospital deaths, the death certificate was used in combination with interviews of next of kin and questionnaires completed by the patients' physicians. Autopsy and coroner reports were also used. For this analysis, CHD was defined as a validated definite or probable MI or definite CHD death through to December 31, 2002. All possible clinical CHD events were reviewed by members of the ARIC Morbidity and Mortality Classification Committee, using published criteria. 22

\section{Statistical Analysis}

Of the 15,792 ARIC participants at baseline, we excluded 329 who had a history of stroke, 1,028 who had a history of CHD or who could not be classified by a history of CHD, and 399 without LDL-C data (192 of these because TG $>400 \mathrm{mg} / \mathrm{dl}$ ). We also excluded, due to small numbers, non- 
Table 2 RH (95\% Confidence Interval) of CHD Incidence With Cigarette Smoking Status According to LDL-C Level

\begin{tabular}{|c|c|c|c|c|c|c|}
\hline & & \multicolumn{5}{|c|}{ LDL-C level $(\mathrm{mg} / \mathrm{dl})$} \\
\hline & & $<100$ & $100-129$ & $130-159$ & $160-189$ & $\geq 190$ \\
\hline \multicolumn{7}{|l|}{ Cigarettes per day } \\
\hline Former smoker & $R H$ & $1.02(0.59-1.76)$ & $1.13(0.79-1.61)$ & $1.19(0.87-1.65)$ & $1.10(0.77-1.58)$ & $1.12(0.72-1.76)$ \\
\hline Never smoked & $R H$ & 1 & 1 & 1 & 1 & 1 \\
\hline Current 1-14 & $R H$ & $1.84(0.91-3.74)$ & $1.76(1.07-2.91)$ & $1.76(1.05-2.93)$ & $1.34(0.72-2.48)$ & $2.07(1.02-4.18)$ \\
\hline Current 15-29 & $R H$ & $1.33(0.66-2.67)$ & $2.09(1.39-3.13)$ & $2.91(2.01-4.21)$ & $2.08(1.37-3.17)$ & $2.25(1.33-3.82)$ \\
\hline Current 30+ & $R H$ & $2.13(0.98-4.61)$ & $2.46(1.43-4.24)$ & $3.80(2.37-6.07)$ & $2.23(1.29-3.85)$ & $3.80(1.98-7.29)$ \\
\hline Slope* & $R H$ & $1.23(0.97-1.57)$ & $1.39(1.19-1.62)$ & $1.61(1.40-1.85)$ & $1.36(1.16-1.59)$ & $1.53(1.26-1.86)$ \\
\hline \multicolumn{7}{|c|}{ Cigarette-years of smoking } \\
\hline Never smoked & $R H$ & 1 & 1 & 1 & 1 & 1 \\
\hline Ever 1-399 & $R H$ & $1.39(0.83-2.35)$ & $1.09(0.75-1.60)$ & $1.19(0.83-1.69)$ & $0.88(0.58-1.33)$ & $1.11(0.69-1.79)$ \\
\hline Ever $400+$ & $R H$ & $1.32(0.78-2.24)$ & $1.79(1.29-2.49)$ & $2.12(1.57-2.85)$ & $1.86(1.33-2.59)$ & $2.06(1.35-3.13)$ \\
\hline Slope $e^{\dagger}$ & $R H$ & $1.15(0.89-1.48)$ & $1.35(1.14-1.60)$ & $1.47(1.26-1.71)$ & $1.39(1.17-1.65)$ & $1.46(1.18-1.80)$ \\
\hline
\end{tabular}

$R H$, relative hazard; CHD, coronary heart disease; $L D L-C$, low-density lipoprotein cholesterol.

*Slope per 1 category increase not including former smokers; ${ }^{\dagger}$ slope per 1 category increase.

RH of CHD incidence for smoking status compared with never smoking adjusted for age, sex, race (African-American, white), education (less than high-school graduate, high-school graduate, greater than high-school graduate), systolic blood pressure (continuous variable), antihypertensive medication (yes, no), triglycerides, high-density lipoprotein cholesterol, ethanol intake, body mass index, waist-to-hip ratio, sports index and diabetes (yes, no).

(a)

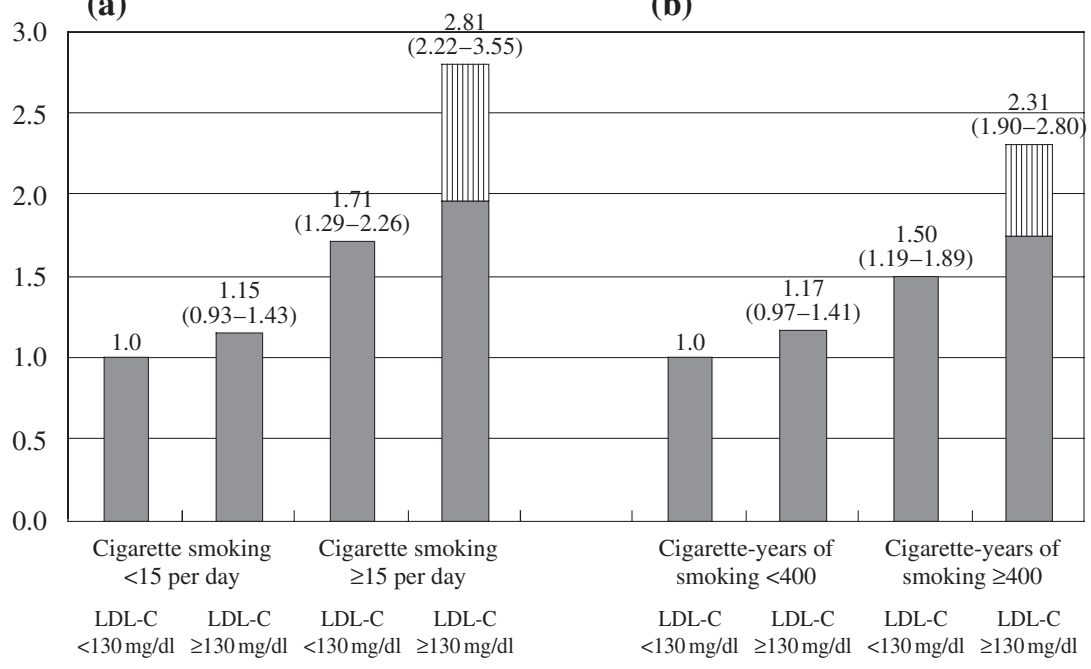

Fig 1. Relative hazard (RH) $(95 \%$ confidence interval) of coronary heart disease incidence by levels of smoking (cutoff points for cigarette smoking were 15 per day (a) or 400 cigarette-years (b) and for low-density lipoprotein cholesterol (LDL-C) the cutoff level was $130 \mathrm{mg} / \mathrm{dl})$. The area with vertical lines shows the excess RH compared with the RH estimated from the product of RHs of high LDL$\mathrm{C}$ and heavy smoking. The $\mathrm{RH}$ were adjusted for age, sex, race (African-American, white), education (less than high-school graduate, high-school graduate, greater than high-school graduate), systolic blood pressure (continuous variable), antihypertensive medication (yes, no), triglycerides, high-density lipoprotein cholesterol, ethanol intake, body mass index, waist-to-hip ratio, sports index and diabetes (yes, no). Fig 1(a) does not include former smokers. white and non-African-American subjects $(\mathrm{N}=42)$. Subjects who did not have complete information on cigarette smoking ( $\mathrm{N}=9)$, did not fast $(\mathrm{N}=451)$ or who were missing other confounding factors $(\mathrm{N}=124)$ were also excluded. In all, 13,410 participants were included in the analysis.

The associations of baseline LDL-C and smoking with other risk factors were assessed using analysis of variance or the chi²-test, as appropriate. Risk factor associations with CHD incidence were estimated from Cox proportional hazard models. RH of CHD for the smoking and LDL-C categories of risk factors were computed using 'never smokers' as the references. In all analyses, we adjusted for age, race (African-American, white), sex, education (less than high-school graduate, high-school graduate, greater than high-school graduate), systolic BP (continuous variable), antihypertensive medication (yes, no), TG, HDL-C, ethanol intake, BMI, WHR, sports index and diabetes (yes, no). Slopes were calculated as the RH of CHD per 1 category increase in smoking. When we calculated the slope for current smoking status, we did not include former smokers in the calculation.

The p-values for multiplicative interactions between LDL-C and smoking status for CHD were usually calcu- lated using LDL-C dichotomized at $130 \mathrm{mg} / \mathrm{dl}$ and dichotomizing smoking status at 15 cigarettes per day or 400 cigarette-years, and by including cross-product terms in the regression models because slopes were higher in heavier smokers and those with higher LDL-C. In tests for interaction involving current smoking status, we ignored the former smokers.

Two-tailed p-values $<0.05$ were considered statistically significant. All statistical analyses were performed using SAS software, version 8.2 (SAS Institute, Cary, NC, USA).

\section{Results}

\section{LDL-C, Smoking and Other Cardiovascular Risk Factors}

The mean value \pm SD of LDL-C was $137 \pm 39 \mathrm{mg} / \mathrm{dl}$ (maximum $504 \mathrm{mg} / \mathrm{dl}$ ). Table 1 shows the association of LDL-C categories with other risk factors for CHD. Mean values of age, systolic BP, TG, WHR and BMI were higher in higher LDL-C categories. Conversely, mean values of HDL-C and ethanol intake were lower in the higher LDL-C categories. The proportion of subjects who were men, diabetic, antihypertensive medication users, and less educated increased across the LDL-C categories. 


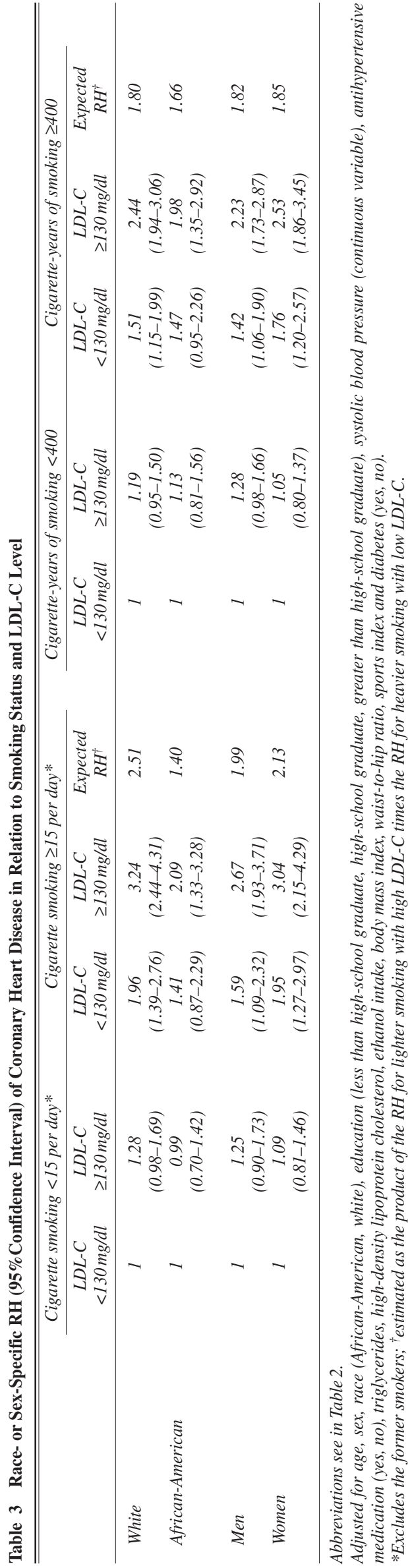

Of the sample, $25 \%$ were current smokers, $32 \%$ were former smokers and $43 \%$ were never smokers. Among current smokers, the mean number of cigarettes smoked per day was $19.1 \pm 10.1$ and 2,390 (70\%) smoked 15 cigarettes or more per day. Compared with never smokers, ever smokers were more often men and were less well educated. Ever smokers had lower BMI, HDL-C and systolic BP, and higher ethanol intake and TG values than never smokers.

\section{Association of Smoking Status With CHD According to $L D L-C$}

Over a mean duration of follow-up of 13.3 years (maximum 16.1 years), 932 incident CHD events occurred. Incidence rates of CHD per 1,000 person-years were 8.38 for current smokers, 5.07 for former smoker and 3.60 for never smokers. Incidence rates also increased across LDL-C groups classified according to NCEP guidelines: $3.28,4.16$, $5.10,7.04$ and 9.38 per 1,000 person-years for optimal $(<100 \mathrm{mg} / \mathrm{dl})$, near optimal/above optimal $(100-129 \mathrm{mg} / \mathrm{dl})$, borderline high $(130-159 \mathrm{mg} / \mathrm{dl})$, high $(160-189 \mathrm{mg} / \mathrm{dl})$ and very high $(\geq 190 \mathrm{mg} / \mathrm{dl})$, respectively.

Table 2 shows the multivariable-adjusted $\mathrm{RH}$ of $\mathrm{CHD}$ incidence for smoking status according to LDL-C category. The slopes of current smoking per 1 smoking category increase were steeper in higher LDL-C groups than in lower LDL-C groups. This was also true when we used cigaretteyears of smoking instead of numbers of cigarettes smoked per day. Similarly, the slope of LDL-C according to smoking status was steeper in heavier smokers 15-29 cigarettes per day, (slope per 1 LDL-C category increase: 1.25; 95\% confidence interval (CI): 1.10-1.41), $\geq 30$ cigarettes per day, (slope per 1 LDL-C category increase: 1.32 ; 95\% CI: 1.11-1.57), than never (slope per 1 LDL-C category increase: 1.15 ; 95\% CI: $1.04-1.28)$ or light smokers $(<15$ cigarettes per day, slope per 1 LDL-C category increase: 1.05; 95\% CI: $0.86-1.27)$. The slope of LDL-C in former smokers was intermediate (slope per 1 LDL-C category increase: 1.22 ; $95 \%$ CI: 1.11-1.35).

When we used $130 \mathrm{mg} / \mathrm{dl}$ as the cutoff for LDL-C and never or current smoking as the cutoff for cigarette smoking, although the RH of current smoking with high LDL-C (RH: 2.88 (95\% CI: 2.25-3.69) was higher than the expected $\mathrm{RH}$ of 2.46, obtained by multiplying the $\mathrm{RH}$ for high LDL with never smoking $(\mathrm{RH}=1.99)$ by current smoking with lower LDL-C $(\mathrm{RH}=1.23))$, the p-value for interaction was not significant $(\mathrm{p}=0.34)$. However, when we used $130 \mathrm{mg} / \mathrm{dl}$ as the cutoff for LDL-C and 15 cigarettes per day as the cutoff for cigarette smoking, the $\mathrm{RH}$ of high smoking exposure with high LDL-C was very high $(\mathrm{RH}=2.81)$ compared with lower smoking and lower LDL$\mathrm{C}(\mathrm{RH}=1.00$, reference) (Fig 1a). This value of 2.81 is higher than the expected RH of 1.97, obtained by multiplying the RH for high LDL with lower smoking $(\mathrm{RH}=1.15)$ by heavy smoking with lower LDL-C $(\mathrm{RH}=1.71)$, p-value for interaction $=0.04$. This finding was also observed when we used 400 cigarette-years of smoking as the cutoff point (Fig 1b), and the p-value for interaction was 0.05 . These relationships were consistently found in men, women, whites, and African-Americans (Table 3); that is, the RH of high smoking exposure with high LDL-C was consistently higher than the expected $\mathrm{RH}$, obtained by multiplying the RH for high LDL-C with lower smoking by heavy smoking with lower LDL-C. 


\section{Discussion}

In this prospective cohort study, the CHD risk associated with heavier smoking and elevated LDL-C were greater than expected from the product of the individual RHs. Although the statistical significance of these interactions was modest, our findings offer support for the hypothesis that the effect of smoking on CHD risk is greater in the presence of an elevated LDL-C level, and conversely, the effect of smoking is less in the presence of a low LDL-C level.

It is well known that incidence rates of CHD among populations with lower cholesterol, such as in eastern Asia and southern Europe in the 1960s, are lower than for contemporary Western populations, despite a higher prevalence of smoking in men.-4 Such differences have led to the belief that smoking is a less important risk factor for CHD in populations with lower cholesterol than in those with higher cholesterol. Well-known factors that differ between higher and lower cholesterol countries are BMI, TC, diet and physical activity ${ }^{24}$ and these might modify the smoking and CHD associations. Therefore, lower blood TC levels and saturated fatty acid intake are leading candidates to explain why CHD rates are low in these populations, despite a high smoking prevalence.

Recent studies that assessed the impact of smoking or cholesterol on different stages of atherosclerosis have revealed that smoking is associated more strongly with advanced, rather than early, subclinical atherosclerosis 5,6 For example, Sharrett et al showed in the ARIC study that the odds ratio of smoking for several stages of atherosclerosis was larger for severe atherosclerosis (lower extremity artery disease or severe carotid artery intimal medial thickness (IMT)) than for moderate IMT? Similar findings were reported by the Multiethnic Study of Atherosclerosis? In contrast, in both those studies LDL-C, relative to smoking, was the more important determinant of the earliest ultrasound-detectable stage of atherosclerosis, in particular, the ultrasound-assessed echolucent lesions indicative of early atheroma with substantial cholesterol-filled cores and minimal fibrous caps? 5 Similarly, a study of young adults reported that the determinants of carotid IMT were only lifetime LDL-C and (inversely) HDL-C, with no substantial associations for smoking pack-years or diabetes, 25 supporting a principal role for LDL-C in early atherosclerosis. Furthermore, the Pathobiological Determinants of Atherosclerosis in Youth (PDAY) Study, which collected information on coronary artery atherosclerosis in persons aged 15-34 years, showed that smokers had a greater prevalence of severe atherosclerosis than non-smokers, ${ }^{26}$ from which those researchers concluded that smoking accelerates the transition from atheroma, with their cholesterol-filled cores, to fibroatheroma, as classified by American Heart Association criteria ${ }^{27}$ Our findings of a multiplicative interaction between smoking and LDL-C may be consistent with the view that smoking accelerates the further progression of cholesterol-filled lesions. It is also possible that the interaction might be explained by other factors that are strongly related to smoking status, such as C-reactive protein, platelet count, leukocyte count ${ }^{28}$ or impaired endothelial dysfunction due to cigarette smoking-induced oxidative stress ${ }^{29}$ Further studies are warranted to clarify the mechanism.

Only Cullen et al, in a smaller study, has previously reported the association of smoking with CHD according to LDL-C levels. Among men aged 40-65 and followed for 8 years 132 major coronary events occurred in 1,444 current smokers and 126 major coronary events in 3,195 never or ex-smokers. Unadjusted RRs of CHD for smokers vs nonsmokers were $2.8,1.7$ and 2.5 within low, middle and high LDL-C tertiles, respectively. Thus, the prior literature does not suggest a multiplicative interaction of smoking with LDL-C or with TC ? $^{-9}$ Our study also did not show a statistically significant interaction when we used 'never smoking' as a cutoff point. However, we found modest but significant interaction when we compared the heavy smokers with light smokers. Our finding suggests that sufficient smoking exposure might be needed to show a significant synergistic effect of LDL-C and smoking on CHD incidence.

The strengths of our study are that it included a large, biracial population-based cohort, careful assessment of CHD incidence over an extended follow-up period, and an ability to adjust our findings for many CHD risk factors. However, even using such a large cohort, the interaction was significant at only modest levels. Studies with even larger numbers of subjects with lower LDL-C may be needed to confirm our hypothesis.

Our data show a much higher risk CHD with heavy cigarette smoking in the presence of higher LDL-C than lower LDL-C, which reinforces the importance of smoking cessation in those with elevated cholesterol. Together with cholesterol reduction, this may synergize in preventing CHD. Furthermore, if we hypothesize that our findings would be applicable to countries with lower cholesterol, such as eastern Asia, where the lifestyle is becoming more westernized $^{30,31}$ and where the prevalence of smoking is still high among men, clinicians should be mindful of a potential rise in CHD incidence. Unless smoking is reduced or LDL-C is appropriately controlled in smokers with higher LDL-C levels, the incidence of CHD in Asian countries may increase dramatically in the future.

\section{Acknowledgments}

The authors thank the staff and participants of the Atherosclerosis Risk in Communities (ARIC) Study for their important contribution.

Dr Hozawa was supported by a grant (14010301) from the Japan Society for the Promotion of Science Research from the Ministry of Education, Culture, Sports, Science and Technology of Japan and the Banyu Fellowship Program sponsored by Banyu Life Science Foundation International.

The ARIC Study is supported by National Heart, Lung, and Blood Institute contracts N01-HC-55015, N01-HC-55016, N01-HC-55018, N01HC-55019, N01-HC-55020, N01-HC-55021, and N01-HC-55022.

\section{References}

1. US Department of Health and Human Services. The health consequences of smoking: A report of the Surgeon General. Atlanta, Georgia: US Depart. of Health and Human Services, CDC, National Center for Chronic Disease Prevention and Health Promotion, Office on Smoking and Health, 2004.

2. Robertson TL, Kato H, Rhoads GG, Kagan A, Marmot M, Syme SL, et al. Epidemiologic studies of coronary heart disease and stroke in Japanese men living in Japan, Hawaii, and California: Coronary heart disease risk factors in Japan and Hawaii. Am J Cardiol 1977; 39: $244-249$.

3. Jee SH, Suh I, Kim IS, Appel LJ. Smoking and atherosclerotic cardiovascular disease in men with low levels of serum cholesterol: The Korea Medical Insurance Corporation Study. JAMA 1999; 282: 2149-2155.

4. Keys A. Seven countries: A multivariate analysis of death and coronary heart disease. Cambridge, MA: Harvard University Press; 1980; $136-160$.

5. Sharrett AR, Coady SA, Folsom AR, Couper DJ, Heiss G; ARIC Study. Smoking and diabetes differ in their associations with subclinical atherosclerosis and coronary heart disease: The ARIC Study. Atherosclerosis 2004; 172: $143-149$.

6. Sharrett AR, Ding J, Criqui MH, Saad MF, Liu K, Polak JF, et al. Smoking, diabetes, and blood cholesterol differ in their associations 
with subclinical atherosclerosis: The Multiethnic Study of Atherosclerosis (MESA). Atherosclerosis 2006; 186: 441-447.

7. Stamler J, Wentworth D, Neaton JD. Is relationship between serum cholesterol and risk of premature death from coronary heart disease continuous and graded? Findings in 356,222 primary screenees of the Multiple Risk Factor Intervention Trial (MRFIT). JAMA 1986; 256: $2823-2828$.

8. Cullen P, Schulte H, Assmann G. Smoking, lipoproteins and coronary heart disease risk: Data from the Münster Heart Study (PROCAM). Eur Heart J 1998; 19: 1632 -1641.

9. Blanco-Cedres L, Daviglus ML, Garside DB, Liu K, Pirzada A, Stamler J, et al. Relation of cigarette smoking to 25 -year mortality in middLe-aged men with low baseline serum cholesterol: The Chicago Heart Association Detection Project in Industry. Am J Epidemiol 2002; 155: $354-360$.

10. The ARIC Investigators. The Atherosclerosis Risk in Communities (ARIC) Study: Design and objectives. Am J Epidemiol 1989; 129: 687-702.

11. Papp AC, Hatzakis H, Bracey A, Wu KK. ARIC hemostasis study. I: Development of a blood collection and processing system suitable for multicenter hemostatic studies. Thromb Haemost 1989; 61: $15-$ 19.

12. Siedel J, Hagele EO, Ziegenhorn J, Wahlefeld AW. Reagent for the enzymatic determination of serum total cholesterol with improved lipolytic efficiency. Clin Chem 1983; 29: 1075-1080.

13. Nagele U, Hagele EO, Sauer G, Wiedemann E, Lehmann P, Wahlefeld $\mathrm{AW}$, et al. Reagent for the enzymatic determination of serum total triglycerides with improved lipolytic efficiency. J Clin Chem Clin Biochem 1984; 22: 165-174.

14. Warnick GR, Mayfield C, Benderson J, Chen JS, Albers JJ. HDL cholesterol quantitation by phosphotungstate- $\mathrm{Mg}^{2+}$ and by dextran sulfate- $\mathrm{Mn}^{2+}$-polyethylene glycol precipitation, both with enzymic cholesterol assay compared with the lipid research method. Am J Clin Pathol 1982; 78: 718-723.

15. Friedewald WT, Levy RI, Fredrickson DS. Estimation of the concentration of low-density lipoprotein cholesterol in plasma, without use of the preparative ultracentrifuge. Clin Chem 1972; 18: 499-502.

16. Expert Panel on Detection, Evaluation, and Treatment of High Blood Cholesterol in Adults. Executive Summary of The Third Report of The National Cholesterol Education Program (NCEP) Expert Panel on Detection, Evaluation, And Treatment of High Blood Cholesterol In Adults (Adult Treatment Panel III). JAMA 2001; 285: 2486-2497.

17. Chambless LE, McMahon RP, Brown SA, Patsch W, Heiss G, Shen YL. Short-term intraindividual variability in lipoprotein measurements: The Atherosclerosis Risk in Communities (ARIC) Study. Am J Epidemiol 1992; 136: 1069-1081.

18. National Heart, Lung, and Blood Institute. Atherosclerosis Risk in Communities (ARIC) Study: Operations manual no. 2: cohort component procedures: Version 1.0. Chapel Hill, NC: ARIC Coordinating Center, School of Public Health, University of North Carolina, 1987.

19. National Heart, Lung, and Blood Institute. Atherosclerosis Risk in
Communities (ARIC) Study: Operations manual no. 11: sitting blood pressure: Version 1.0. Chapel Hill, NC: ARIC Coordinating Center, School of Public Health, University of North Carolina, 1987.

20. Baecke JA, Burema J, Frijters JE. A short questionnaire for the measurement of habitual physical activity in epidemiological studies. Am J Clin Nutr 1982; 36: 936-942.

21. National Heart, Lung, and Blood Institute. Atherosclerosis Risk in Communities Study (ARIC): Operations manual no. 5: Electrocardiography: Version 1.0. Chapel Hill, NC: ARIC Coordinating Center, School of Public Health, University of North Carolina, 1987.

22. White AD, Folsom AR, Chambless LE, Sharret AR, Yang K, Conwill D, et al. Community surveillance of coronary heart disease in the Atherosclerosis Risk in Communities (ARIC) Study: Methods and initial two years' experience. J Clin Epidemiol 1996; 49: 223 233.

23. Prineas RJ, Crow RS, Blackburn H. The Minnesota code manual of electrocardiographic findings: Standards and procedures for measurement and classification: Littleton, MA: John Wright, 1982.

24. Watanabe H, Yamane K, Fujikawa R, Okubo M, Egusa G, Kohno N. Westernization of lifestyle markedLy increases carotid intima-media wall thickness (IMT) in Japanese people. Atherosclerosis 2003; 166: $67-72$.

25. Davis PH, Dawson JD, Riley WA, Lauer RM. Carotid intimal-medial thickness is related to cardiovascular risk factors measured from childhood through middLe age: The Muscatine Study. Circulation 2001; 104: 2815-2819.

26. Zieske AW, McMahan CA, McGill HC Jr, Homma S, Takei H, Malcom GT, et al. Smoking is associated with advanced coronary atherosclerosis in youth. Atherosclerosis 2005; 180: 87-92.

27. Stary HC, Chandler AB, Dinsmore RE, Fuster V, Glagov S, Insull W $\mathrm{Jr}$, et al. A definition of advanced types of atherosclerotic lesions and a histological classification of atherosclerosis: A report from the Committee on Vascular Lesions of the Council on Arteriosclerosis, American Heart Association. Arterioscler Thromb Vasc Biol 1995; 15: $1512-1531$.

28. Yasue H, Hirai N, Mizuno Y, Harada E, Itoh T, Yoshimura M, et al. Low-grade inflammation, thrombogenicity, and atherogenic lipid profile in cigarette smokers. Circ J 2006; 70: 8-13.

29. Tanriverdi H, Evrengul H, Kuru O, Tanriverdi S, Seleci D, Enli Y, et al. Cigarette smoking induced oxidative stress may impair endothelial function and coronary blood flow in angiographically normal coronary arteries. Circ J 2006; 70: 593-599.

30. Zhang X, Patel A, Horibe H, Wu Z, Barzi F, Rodgers A, et al; Asia Pacific Cohort Studies Collaboration, et al (Asia Pacific Cohort Studies Collaboration). Cholesterol, coronary heart disease, and stroke in the Asia Pacific region. Int J Epidemiol 2003; 32: 563-572.

31. Okayama A, Ueshima H, Marmot MG, Elliott P, Yamakawa M, Kita Y. Different trends in serum cholesterol levels among rural and urban populations aged 40-59 in Japan from 1960 to 1990. J Clin Epidemiol 1995; 48: 329-337. 